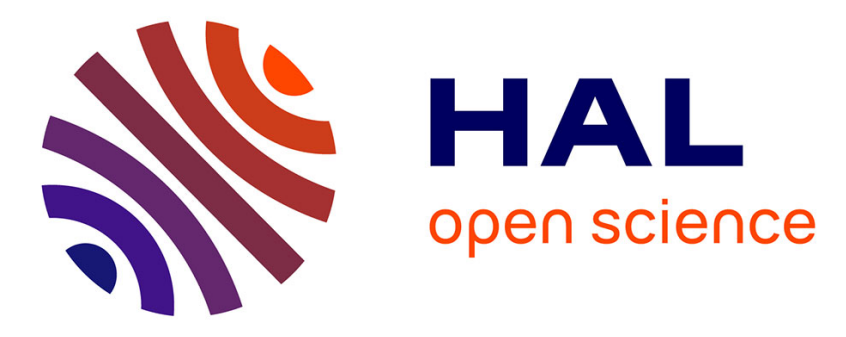

\title{
Sensitivity of optical correlation to color change of target images
}

\author{
Marwa El Bouz, Ayman Alfalou, Christian Brosseau, M.S. Alam, Sofia Qasmi,
} Yousri Ouerhani

\section{- To cite this version:}

Marwa El Bouz, Ayman Alfalou, Christian Brosseau, M.S. Alam, Sofia Qasmi, et al.. Sensitivity of optical correlation to color change of target images. Optical Pattern Recognition XXIII, Apr 2012, Baltimore, United States. pp.839801. hal-00783036

\section{HAL Id: hal-00783036 https://hal.science/hal-00783036}

Submitted on 31 Jan 2013

HAL is a multi-disciplinary open access archive for the deposit and dissemination of scientific research documents, whether they are published or not. The documents may come from teaching and research institutions in France or abroad, or from public or private research centers.
L'archive ouverte pluridisciplinaire $\mathbf{H A L}$, est destinée au dépôt et à la diffusion de documents scientifiques de niveau recherche, publiés ou non, émanant des établissements d'enseignement et de recherche français ou étrangers, des laboratoires publics ou privés. 


\title{
Sensitivity of optical correlation to color change of target images
}

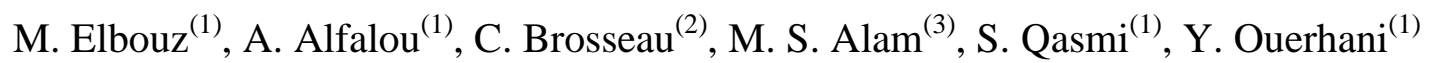 \\ ( ${ }^{1)}$ ISEN Brest, Groupe Vision, L@ bISEN, 20 rue Cuirassé Bretagne, CS 42807, \\ 29228 Brest Cedex 2, France.

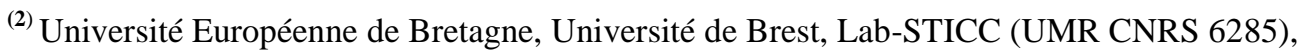 \\ CS 93837, 6 avenue Le Gorgeu, 29238 Brest Cedex 3, France. \\ ${ }^{(3)}$ Department of Electrical and Computer Engineering, EEB 75, University of South Alabama, \\ 6001 USA South Dr., Mobile, AL 36688-0002, USA.
}

\begin{abstract}
Optical correlation is based on the comparison of contours between an unknown target image and a known reference image. However, it does not usually include the color image information in the decision making process. In order to render the correlation method sensitive to color change, we propose a general method based on the decomposition of the target image in its three color components using, either the normalized RGB (red, green, blue) color space, or the normalized HSV (hue, saturation, value) space. Then, the correlation operation is carried out for each color component and the results are merged in order to make a decision. The aforementioned steps can alleviate some of the problems associated with illumination changes in the target image but do utilize color information of the target image. To overcome these problems, we propose to convert the color information in contour information into a signature corresponding to the color information of the target image. This technique and test results are presented to validate its effectiveness. The preliminary results obtained with this technique are encouraging.
\end{abstract}

Keywords: correlation, VLC correlator, POF filter, composite filter, segmented composite filter, color pattern recognition, face recognition.

\section{INTRODUCTION}

In the context of pattern recognition, there have been great efforts to develop architectures and applications over the past decades. Optical and numerical architectures were proposed to deal with this issue. In this work, we shall consider more specifically the former ones. An optical correlator (OC) is a device for comparing a target image (image to be recognized) with a reference image of a database. Among the many OC proposed in the literature, the joint transform correlator (JTC) [1] and the VanderLugt correlator (VLC) [2] have engendered considerable interest. The latter was selected for studying the sensitivity of OC to color change of target images. The vast majority of studies dealing with VLC dealt with the fabrication of correlation filters [2], e.g. phase-only filters (POF) [3], amplitude modulated POF (AMPOF) [4]. While POF and AMPOF have a high discrimination capability compared to the standard adapted filter and is adapted for edge detection, they cannot deal with the color information of the target image. Additionally, AMPOF is characterized by a low robustness against changes (rotation, scale) of the reference image. It is worth mentioning that Iftekharuddin et al. [5] have suggested an optimized version of OC to deal with rotation. Other types of filters were proposed to increase the performances of the VLC, e.g. MACH [6], MACE [7], and composite [8]. The latter was shown to have a good compromise between discrimination and robustness, decreasing the number of correlations to make a reliable decision. An optimized version of such filter was recently proposed by Alfalou et al. [9] allowing them to significantly increase the number of references to be merged in such filter. However, the main drawback of this solution is to generate isolated pixels which yield to low overall performances of this kind of filter. Next, Al Falou and Brosseau [10] have proposed a new type of optimized composite filter, i.e. the ASPOF. These authors found useful to introduce a new segmentation criterion to characterize the correlation plane of this filter. Each of the stages has various parameters that must be optimized in order to increase the robustness and discriminating ability while increasing the number of reference images contained in the filter. However, all these filters are not adapted to deal with the color image information. 
Of particular interest has been the correlation between color images. For that purpose, Alam et al. [1113] suggested to use an adaptation of the JTC. Recently, we have also proposed to use a segmented composite filter to deal with this issue [14]. However, this method has a low discrimination capability and is very sensitive to color change of target images. Here, we show that a numerical approach is well adapted to accomplish our correlation purpose. Generally speaking, numerical methods allow increasing the performances of OC, e.g. independent component analysis (ICA) method for image encryption [15] and face recognition [16].

$\mathrm{OC}$ is based on the comparison of contours between an unknown target image and a known reference image. However, it does not include the color image information in the decision making process. To overcome this problem, we propose to convert the color information in contour information into a signature corresponding to the color information of the target image. A numerical preprocessing based on a new coding of the color images is suggested. Each color will be assigned a specific pattern according its intensity. Two color spaces will be considered, i.e. RGB and HSV (hue, saturation, value). This technique and test results are presented to validate its effectiveness. The preliminary results obtained with this technique are encouraging.

\section{COLOR RECOGNITION METHOD}

We begin by recalling the principle of the color correlation-based technique proposed in a previous work [14]. Next, we suggest improving this technique by considering the normalized RGB and HSV color spaces. Fig. 1 illustrates the principle of this method. The RGB or HSV decomposition of the target image was first realized, e.g. Fig. 1 illustrates the case of the RGB decomposition. Each component of the target image is introduced in the VLCs (Fig. 1). Each correlator utilizes a filter which depends of the input component. A correlation plane for each component is obtained at the output of the VLCs. Then, the decision is made.

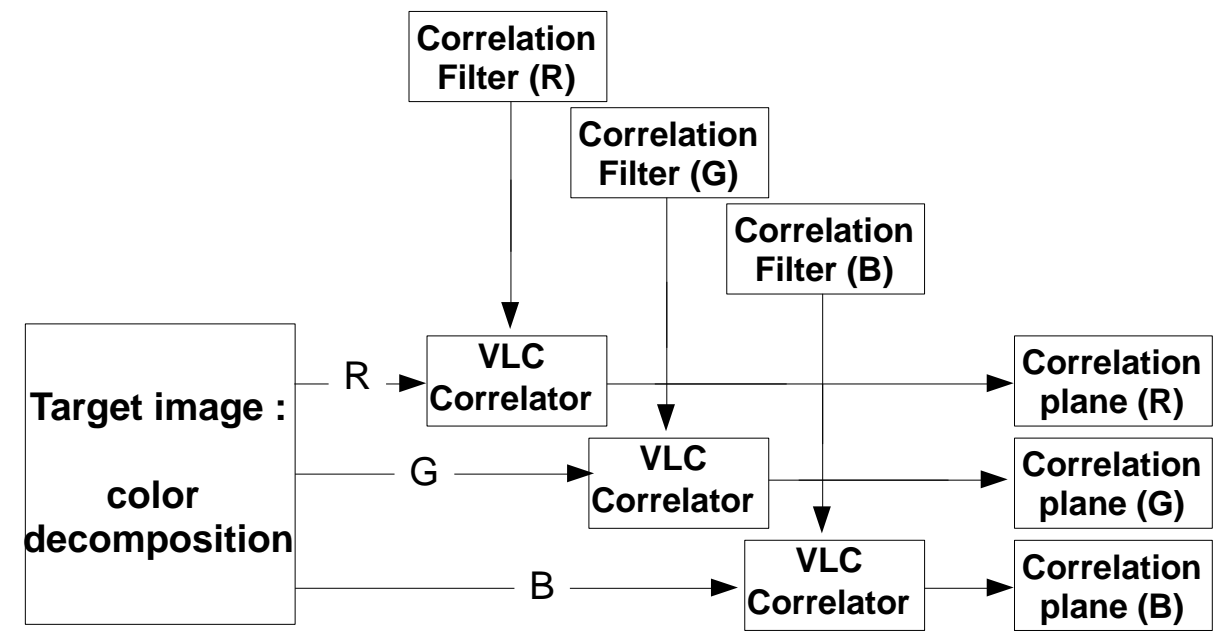

Fig. 1. Illustrating the principle of color recognition method.

The first tests using a POF and the two color spaces indicate that this method is not effective for OC of color images since it remains too sensitive to the illumination changes in the target image with respect to the reference image. In addition, we noted that it leads to discrimination performances. To overcome these problems, the following normalized color spaces were used

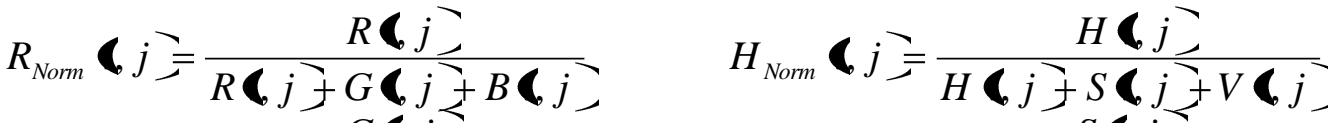

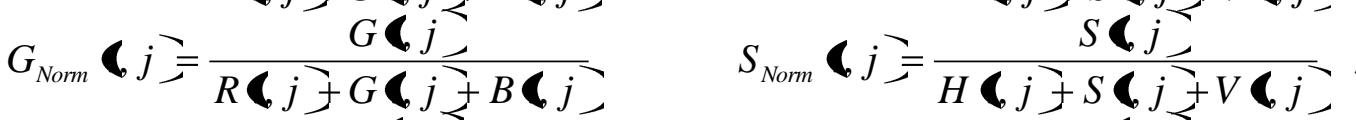

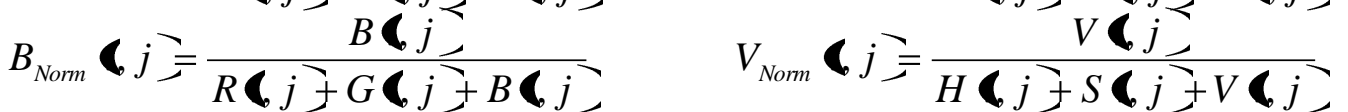

where $H_{\text {Norm }}, S_{\text {Norm }}$ and $V_{\text {Norm }}$ are the normalized versions of $H, S$ and $V$ (same notation for RGB). We found that the correlation results obtained with the RGB color space remained sensitive to illumination conditions. This can strongly impact the decisions, especially if these conditions differ strongly between the target and reference 
images. In contrast, a lower sensitivity to illumination conditions is obtained when the $H S V_{\text {Norm }}$ color space is used. This is illustrated in Fig. 2. Fig. 2 (a) shows the correlation planes of the target image with three correlation filters fabricated with a reference image which is identical to the target image. In every case a narrow correlation peak can be observed which is consistent to what we expect with the use of a POF. Correlation results using the $H S V_{\text {Norm }}$ color space are shown in Fig. 2 (b) when the illumination conditions of the target and reference images are different. As can be seen, the correlation peaks are strongly noised. Unfortunately, we also found that the discrimination capability is low when there is a color change in the target image with respect to the reference

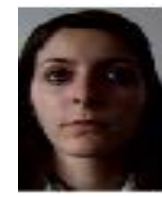

Target image

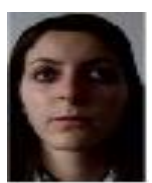

Reference image

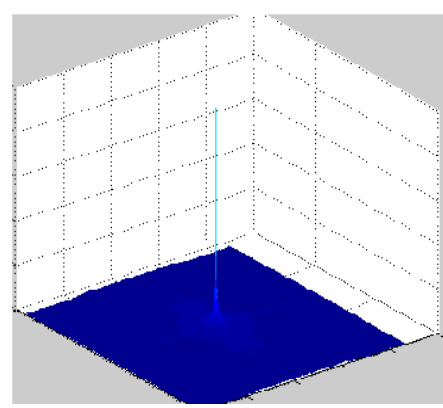

Correlation plane $(\mathrm{H})$

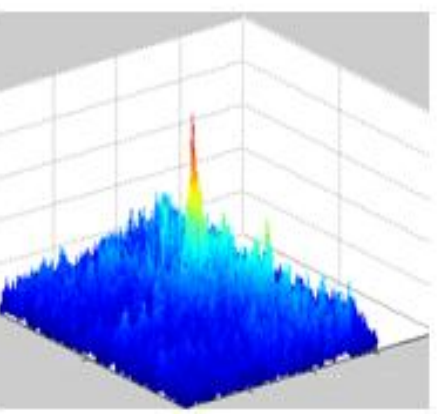

Correlation plane $(\mathrm{H})$

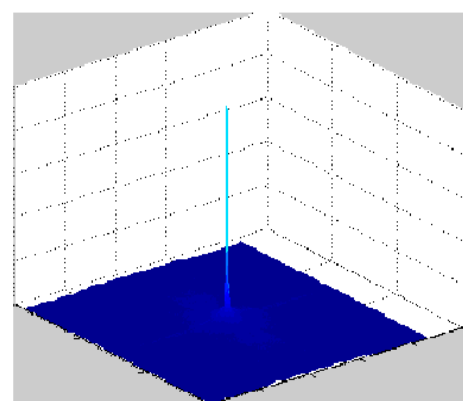

Correlation plane (S)

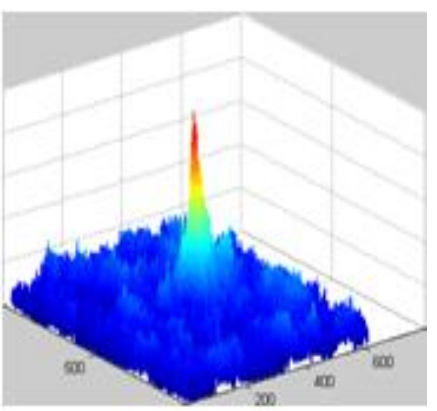

Correlation plane (S)

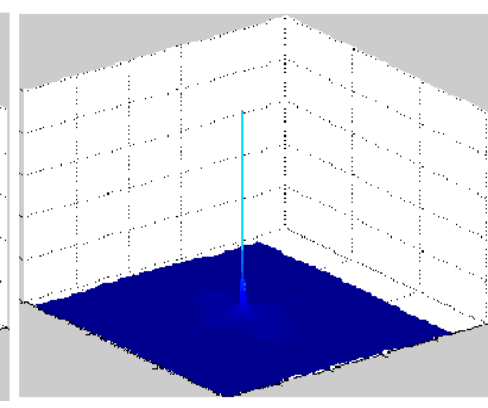

Correlation plane (V)

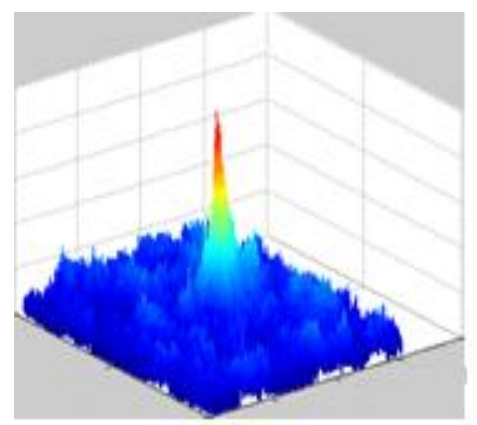

Correlation plane (V) (a)

(b)

Fig. 2. Correlation results between two (a) identical images, and (b) identical images with different illumination conditions.

\section{METHOD}

Here we report a new correlation-based method which is highly discriminating against color changes of target images. For this purpose, red spots with different surface areas were added to the face color image displayed in Fig. 3 (a). The results are illustrated in Fig. 3 (b)-(e).

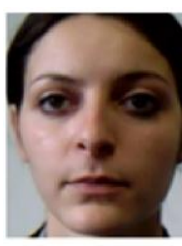

(a)

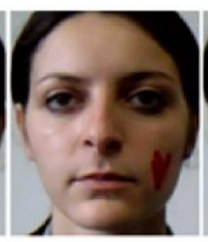

(b)

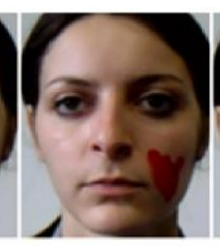

(c)

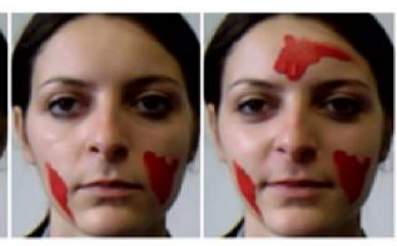

(d)

(e)

Fig. 3. The database employed in this study. 
The implementation of the procedure is as follows. The RGB or HSV decomposition of the target image is first realized. Then, each pixel is converted in contour according a specific signature, i.e. a pattern selected in a base (Fig. 4), which depends of the color intensity of the pixel and the color space. Two requirements of the method need to be emphasized: (1) each change of color should be related to a given pattern even for small spots, and (2) a great variety of patterns should be used in order to minimize shape correlation. Fig. 4 shows the base of 26 patterns used in this study. Three palettes, one per component, were considered, e.g. Fig. 5 shows an example for the red color.

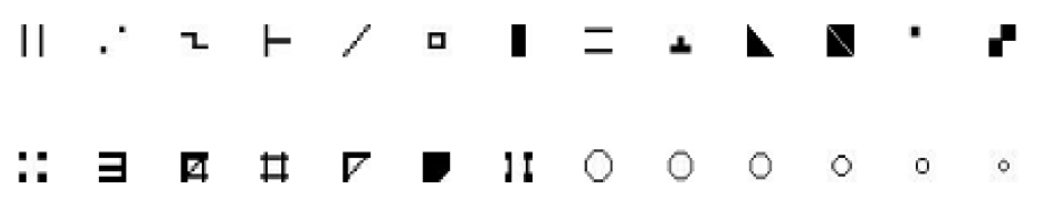

Fig. 4. Base of the 26 patterns.

It is worth mentioning that the choice of the color palettes is important since it will impact the discrimination capability of the method.

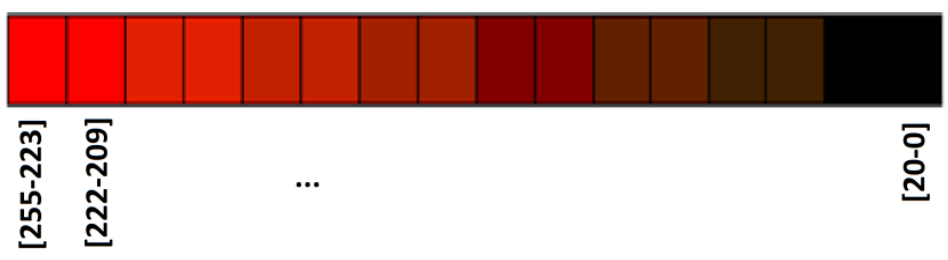

Fig. 5. An example of color palette for red color.

Many color palettes were tested. Those which were used in this study are:

$\mathrm{R}: \quad[0,0.156] ;[0.16,0.313] ;[0.317,0.372] ;[0.376,0.627] ;[0.631,0.690] ;[0.694,0.815] ;[0.819,0870] ;[0.874,1]$.

$\mathrm{V}: \quad[0,0.125] ;[0.129,0.313] ;[0.317,0.435] ;[0.439,0.564] ;[0.568,0.690] ;[0.694,0.815] ;[0.819,0.931] ;[0.941,1]$.

B : $\quad[0,0.125] ;[0.129,0.372] ;[0.376,0.627] ;[0.631,0.874] ;[0.878,1]$. Once the color palettes are specified, the correspondance with patterns can be done. We employed large patterns, i.e. $16 \times 16$ pixels. Fig. 6 illustrates such coding protocol. Fig. 6 (a) shows the target image with 3 red spots without coding; Fig. 6 (b) shows its red component which is coded with a 8-level palette; Fig. 6 (c) presents its green component which is coded with a 8-level palette, and Fig. 6 (d) shows its blue component with a 5-level palette.

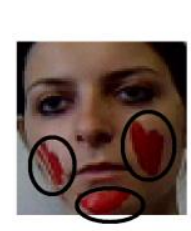

(a)

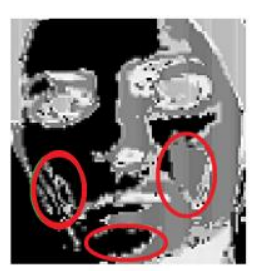

(b)

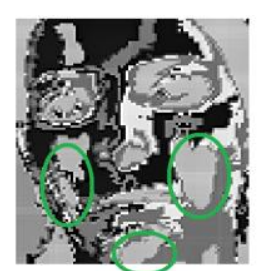

(c)

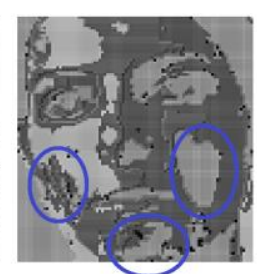

(d)

Fig. 6. An example of color coding.

Once the coding of the images is performed, they are ready for correlation according the scheme displayed in Fig. 1. Correlation results are presented in Fig. 7. Autocorrelation of the face shown in the left column, first row with itself is shown in the correlation plane of the right column, first row of Fig. (a). Similarly, the planes obtained by correlating the other images and the face shown in the first column, first row are illustrated in Fig. 7 (b)-(d). To determine the degree of similarity between the target and reference images, we used the peak-tocorrelation energy (PCE) efficiency criterion [2]. 

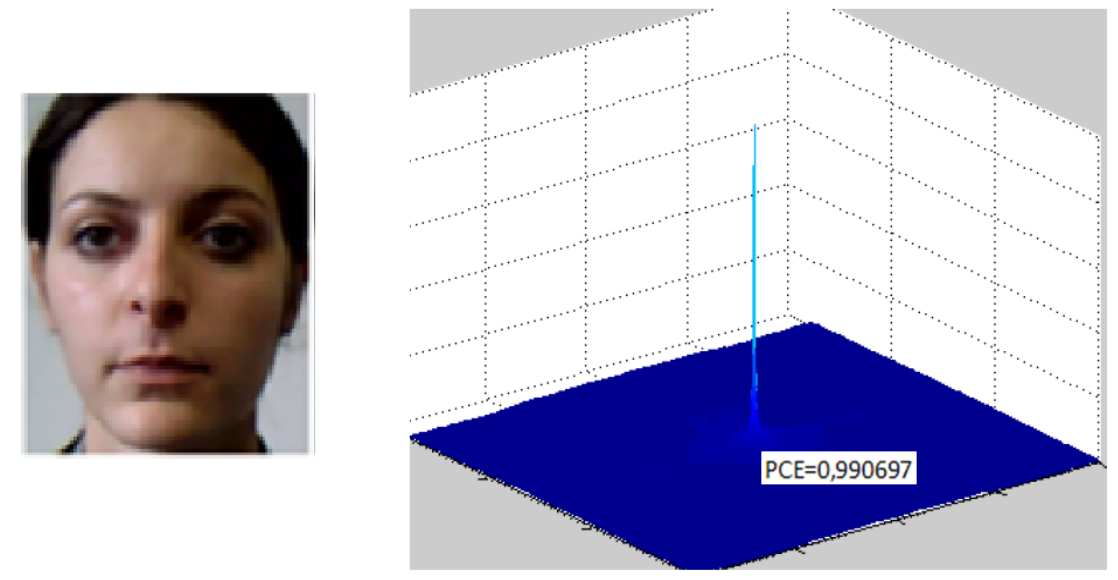

(a)
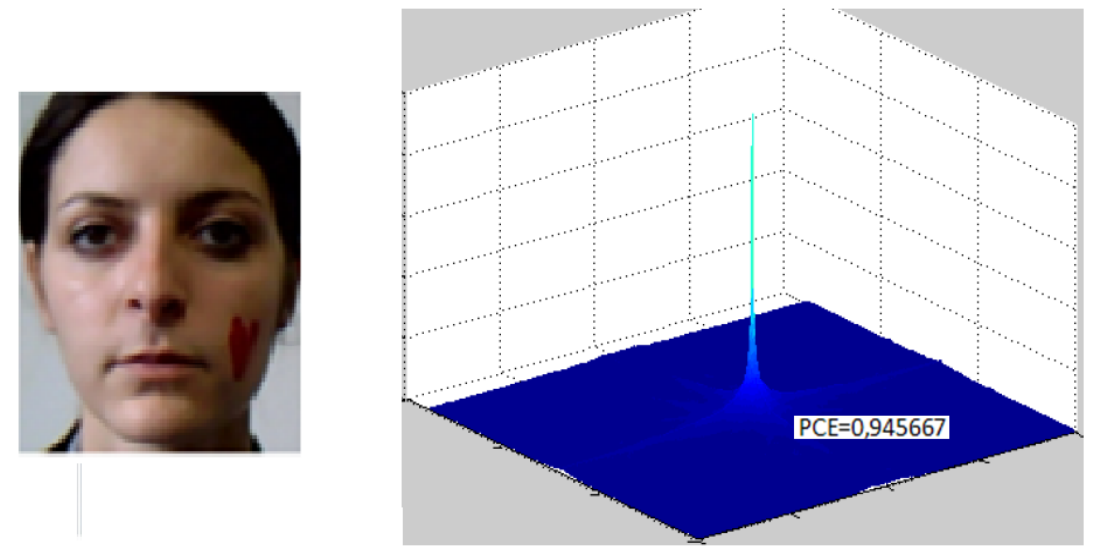

(b)
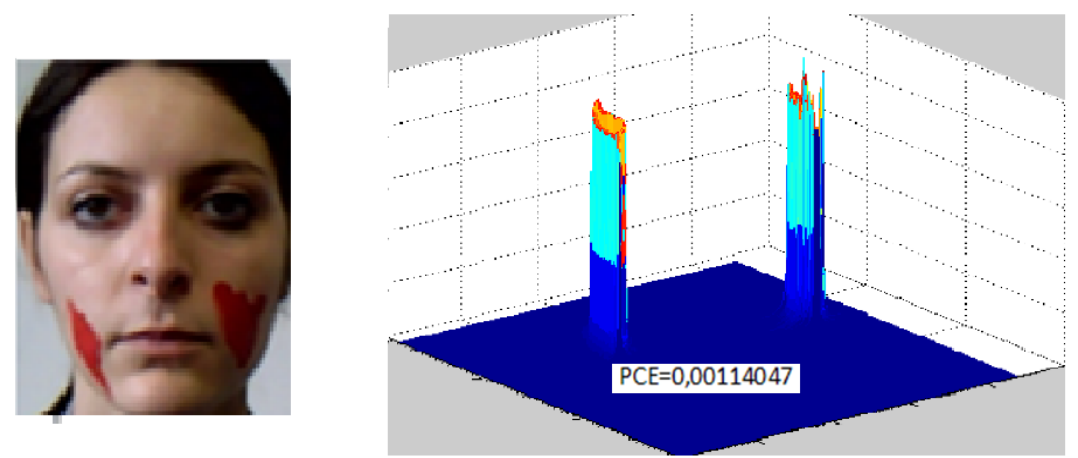

(c)
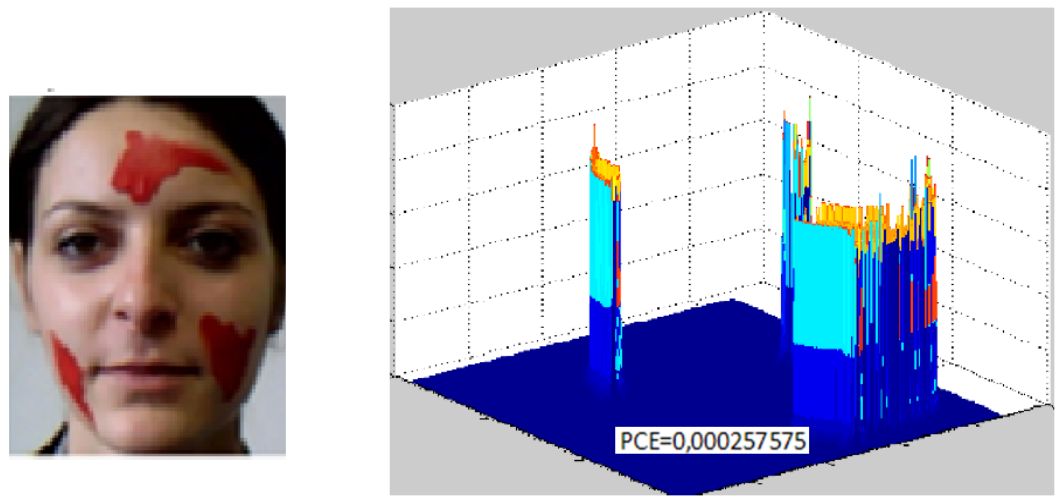

(d)

Fig. 7. Correlation results obtained with our color coding.

Fig. 7 illustrates the good discriminating power of the technique. A significant decrease of the PCE values is observed when comparing Fig. 7 (a) and Fig. 7 (b) even if the red spot has a small extent. What is noticeable is 
that no peak can be observed when correlating the images displayed in the left column, third row (Fig. 7 (c)) and fourth row (Fig. 7 (d)), with the reference (Fig. 7 (a)).

\section{CONCLUSION}

In summary, we have investigated a correlation-based technique of color images with the purpose of decreasing its sensitivity to the illumination conditions of the target image with respect to the reference image. This issue can be addressed by using the normalized HSV color space. Our preliminary results are encouraging since they allowed us to discriminate between two images having the same pattern but differing with a color variation over a small surface compared to the image size. This has been made possible by converting the color information in contour information with a signature corresponding to the color information in the target.

\section{Acknowledgments}

We would like to thank M. Al Sayed and S. Sid-Ahmed for helpful discussions.

\section{References}

[1] Yu F. T. S., Jutamulia S., Optical Pattern Recognition, (Cambridge University Press, Cambridge, 1998).

[2] Alfalou A. and Brosseau C., "Understanding Correlation Techniques for Face Recognition: From Basics to Applications," Face Recognition Book, Milos Oravec (Ed.), ISBN: 978-953-307-060-5, INTECH, Available from: http://sciyo.com/articles/show/title/understanding-correlation-techniques-for-face-recognition-from-basicsto-applications, 354-380 (2010). [3] J. L. Horner and P. D. Gianino, "Phase-only matched filtering," Appl. Opt. 23, 812-816 (1984).

[4] Awwal A. A. S., "What can we learn from the shape of a correlation peak for position estimation?" Appl. Opt. 49, B40-B50 (2010).

[5] Iftekharuddin K. M., Ahmad F., and Karim M. A., "Rotation invariant target recognition using amplitude coupled minimum average correlation energy filter", Opt. Eng. 35, 1009-1014 (1996).

[6] Zhou H.and Chao T.- H., "MACH filter synthesizing for detecting targets in cluttered environment for grayscale optical correlator,” Proc. SPIE 3715, 394 (1999).

[7] Mahalanobis A., Vijaya Kumar B. V. K., and Casassent D., "Minimum average correlation energy filters," Appl. Opt. 26, 3633-3640 (1987).

[8] Vijaya Kumar B. V. K., “Tutorial survey of composite filter designs for optical correlators,” Appl. Opt. 31, 4773-4801 (1992).

[9] Alfalou A., Keryer G., and de Bougrenet de la Tocnaye J. L., "Optical implementation of segmented composite filtering," Appl. Opt. 38, 6129-6135 (1999).

[10] Alfalou A.and Brosseau C., "Exploiting root-mean-square time-frequency structure for multiple-image optical compression and encryption," Opt. Lett. 35, 1914-1916 (2010).

[11] Alam M. S., Goh S. F., and Dacharaju S., "Three-dimensional color pattern recognition using fringeadjusted joint transform correlation with CIELab coordinates," IEEE Trans. Instr. Meas. 58, 2176 (2010).

[12] Islam M. N., Alam M. S., and Haider M. R., "Class-associative color pattern recognition using shifted phase-encoded joint transform correlation,” Opt. Eng. 45, 075006 (2006).

[13] Alam M. S. and Wai C. N., "Color pattern recognition using fringe-adjusted joint transform correlation," Opt. Eng. 40, 2407 (2001).

[14] Soualmi S., Alfalou A., Hamam H., Bensrhair A., "Pattern recognition based on single-channel correlation of color images". COIN-NGNCON 2006, Joint International Conferences on Optical Internet and Next Generation Network, MoC2-7, IEEE Catalog Number: 06EX1473, ISBN: 89-955301-4-6 98560, 254-258, (2006).

[15] Alfalou A.and Mansour A., "All optical video-image encryption enforced security level using ICA". J. Opt. A : Pure Appl. Opt. 9, 787-796 (2007).

[16] Alfalou A. and Brosseau C., "Robust and discriminating method for face recognition based on correlation technique and independent component analysis model," Opt. Lett. 36, 645-647 (2011). 